\section{Resting pulse rates in a glaucoma clinic: the effect of topical and systemic beta- blocker usage}

C Tattersall, S Vernon and R Singh

\begin{abstract}
Introduction Beta-blockers have, for 25 years, been a commonly used agent in the treatment of raised intraocular pressure (IOP). Betablockers can also reduce the pulse rate. With no available literature examining a cohort of patients, this study aims to investigate the resting pulse rates in patients attending a specialist glaucoma clinic in order to identify if routine review of ophthalmic medication use is indicated.

Method The resting pulse rates of patients attending a glaucoma clinic were measured using pulseoximetry, with a medical and drug history established for each patient.

Results In all, 205 patients were included in the study. A total of 101 (49\%) of patients were using beta-blockers in some form. The mean pulse rate for patients not using beta-blockers (104 patients) was 76 beats per minute (bpm), for topical use only (68 patients) it was
\end{abstract} $70.3 \mathrm{bpm}$, for oral use (18 patients) it was $64.7 \mathrm{bpm}$, and $58 \mathrm{bpm}$ for patients using both topical and oral beta-blockers (15 patients).

Groups using beta-blockers (oral, topical, oral and topical) were considered in relation to patients not using beta-blockers. All groups using beta-blockers showed a significant association with causing a bradycardia of less than $60 \mathrm{bpm}$. Patients with a pulse rate of less than $50 \mathrm{bpm}$ were significantly more likely to be using topical and oral beta-blockers than oral beta-blockers alone $(P=\mathbf{0 . 0 1})$.

Conclusion Topical beta-blockers should be used with caution, even in the presence of established systemic beta-blocker use. Routine pulse rate monitoring and review of ophthalmic medication are indicated in patients using beta-blocker therapy. Eye (2006) 20, 221-225. doi:10.1038/sj.eye.6701859; published online 1 April 2005

Keywords: glaucoma; pulse; beta-blocker

\section{Introduction}

Topical beta-blockers have a well-established role in the reduction of intraocular pressure, ${ }^{1,2}$ however, their side effects are also well documented, potentially affecting both cardiac ${ }^{3-8}$ and respiratory functions. ${ }^{3,4,9-11}$ In recent years new agents such as prostaglandin analogues have been developed with a better safety profile, as well as comparable efficacy, ${ }^{12-15}$ leading to beta-blockers not now necessarily being the first-line treatment in glaucoma. However, for many ophthalmologists, betablockers remain in frequent use, as either a primary treatment or as an adjunct to other agents.

The scientific literature reveals very little with regard to the effect of the concomitant use of topical and oral beta-blockers in relation to resting pulse rates. All studies reviewed used either a very small sample size, were subject to a strict inclusion/exclusion criteria, ${ }^{16}$ were reviews, ${ }^{17}$ or were individual case reports. ${ }^{18,19}$ We therefore report the results of a study of resting pulse rates in patients attending a hospital-based glaucoma clinic, in order to identify if any individuals required a reevaluation of their ophthalmic medication based upon resting pulse rate alone.

\section{Method}

The routine monitoring of resting pulse rates in glaucoma patients was introduced during August 2003 in one glaucoma clinic. Pulse rates were measured using a hospital maintained handheld pulseoximeter (Nellcor NPB-40) attached to the index finger of patients for at least $3 \mathrm{~min}$. Pulseoximetry was conducted after
Department of

Ophthalmology, Queens Medical Centre, NHS trust, Nottingham, UK

Correspondence: C Tattersall, Department of Ophthalmology, Queens Medical Centre, NHS trust, Derby Road, Nottingham NG7 2UH, UK Tel: +441159249924 ext. 35651;

Fax: + 441159194486 E-mail: chris.tattersall@ mail.qmcuh-tr.trent.nhs.uk

Received: 25 November 2004

Accepted in revised form: 8 February 2005; Published online: 1 April 2005 
the patient had rested in a seated position for at least $10 \mathrm{~min}$, following an explanation of the procedure. All patients were measured, including those not currently using beta-blockers. Recordings taken during the first 2 months of this new practice were included in the study. Information was elicited from the patient and medical notes, with regard to all medications currently prescribed as well as the patients' medical history. Patients who were found to have a pulse rate of less than $50 \mathrm{bpm}$ were offered an ECG, which was then reviewed by a cardiologist. These patients, along with any patient having a pulse rate of over $100 \mathrm{bpm}$ were referred to their general practitioner.

\section{Results}

In all, 208 patients were assessed over a period of 2 months. For analysis, patients were separated into one of four groups, these groups being:

- Patients not using any beta-blockers.

- Patients using topical beta-blockers only.

- Patients using oral beta-blockers only.

- Patients using both topical and oral beta-blockers (dual therapy).

As three patients $(1.4 \%)$ were taking a pulse rate reducing medication which was not a beta-blocker (Digoxin), these patients were excluded from the study. In all, 205 patients were therefore used in the analysis.

The mean age of all patients was 72.55 (SD 12.2), with no significant difference $(P=0.28)$ between the ages of patients on beta-blockers (mean 72.19, SD 10.92) and those not using beta-blockers (mean 72.95, SD 13.37).

In all, $101(49 \%)$ of patients were using beta-blockers in some form. Of these 68 were using topical only, 18 used oral only, and 15 used dual therapy. In patients using topical medication, the breakdown of use was as follows: $83(48.5 \%)$ of patients were using a topical beta-blocker, with 122 (71\%) using a Prostaglandin Analogue. In all, 43 (25\%) of patients were using both agents (Figure 1).

The mean pulse rate for patients not using betablockers was 76 beats per minute (bpm) (SD 13), for topical use only it was $70.3 \mathrm{bpm}$ (SD 11), for oral use it was $64.7 \mathrm{bpm}$ (SD 10.6), and $58 \mathrm{bpm}$ (SD 10) for patients using dual therapy. The spread of pulse rate values are shown as a boxplot in Figure 2.

Groups were compared, using a one-way ANOVA test in order to identify any significant differences in pulse rates between the groups. This proved highly significant $(P<0.0001)$. Following this result, the significance between the individual groups using beta-blockers when compared to the group with no beta-blockers was assessed using an unpaired $t$-test (Table 1).

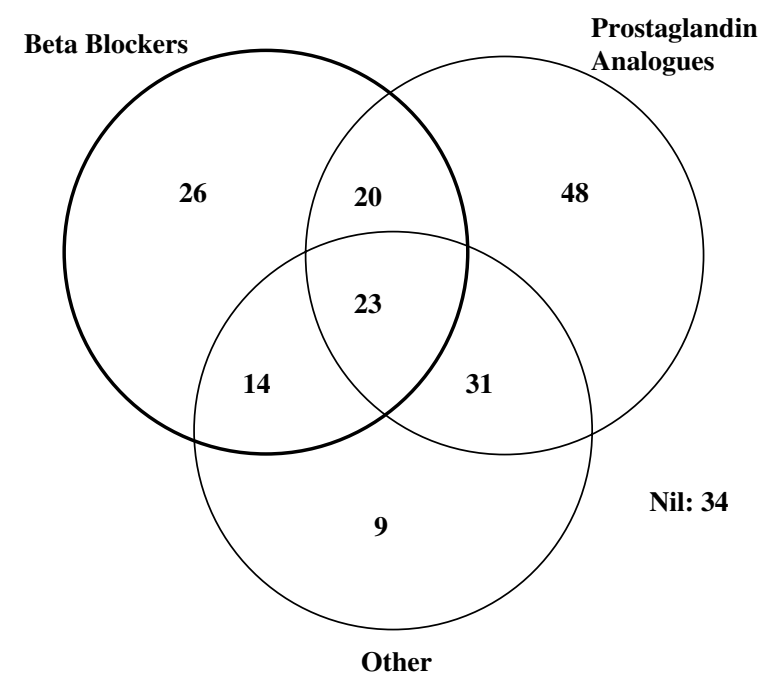

Figure 1 All topical medication used in the cohort (count).

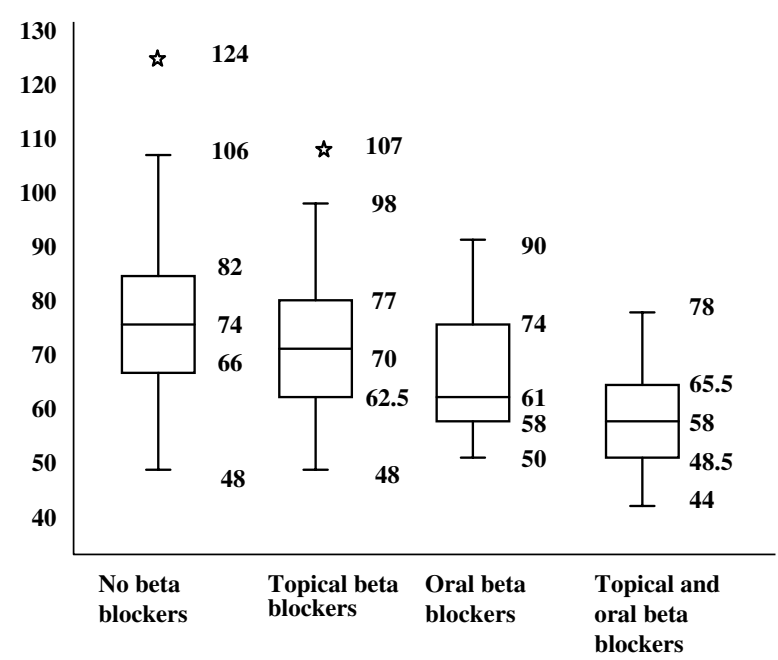

Figure 2 Pulse rates.

Table 1 Differences between heart rates ( $t$-test)

\begin{tabular}{lc}
\hline No beta-blockers vs & Significance $(\mathrm{P})$ \\
\hline Topical only & 0.003 \\
Oral only & 0.0007 \\
Dual therapy & $<0.0001$ \\
\hline
\end{tabular}

Taking an accepted value for bradycardia as below $60 \mathrm{bpm},{ }^{20}$ Fisher's test was used in order to identify any association between bradycardia and each specific group. Groups using beta-blockers (oral, topical, dual) were considered individually in relation to patients not using beta-blockers. All groups using beta-blockers showed a significant association with bradycardia (Table 2). 
Table 2 Association with bradycardia $<60$ bpm (Fisher's test)

\begin{tabular}{lc}
\hline No beta-blocker vs & Significance $(\mathrm{P})$ \\
\hline Topical only & 0.0008 \\
Oral only & 0.0007 \\
Dual therapy & 0.0001 \\
\hline
\end{tabular}

Table 3 Comparison with dual therapy (Fisher's test)

\begin{tabular}{lcc}
\hline & P-value at $<60 \mathrm{bpm}$ & P-value at $<50 \mathrm{bpm}$ \\
\hline Topical only & 0.02 & 0.0006 \\
Oral only & 0.5 & 0.01 \\
\hline
\end{tabular}

As the only group whose mean pulse rate was below $60 \mathrm{bpm}$ was the dual therapy group, the concomitant effect of topical and oral beta-blockers was considered. Fisher's test was employed to examine whether an association with bradycardia existed when comparing the use of dual beta-blockers vs oral only and topical only. When dual therapy was compared to topical only there existed, as expected, a significant association with bradycardia at both the $<60$ and $<50$ bpm levels of pulse rate. A highly important result however was that significance was shown in comparing oral only to dual therapy at the $<50 \mathrm{bpm}$ level, showing the influence of topical medication at this level of bradycardia (Table 3).

All patients $(n=5)$ who showed a pulse rate of less than $50 \mathrm{bpm}$ accepted the offer of an ECG. In three of the five patients a bradycardia of $50 \mathrm{bpm}$ or less was confirmed. One of these five patients was considered to have evidence of borderline 1st degree A-V block in which beta-blocker use may have been a contributory factor. In this case, the patient's GP opted to discontinue the systemic beta-blocker.

\section{Discussion}

Topical beta-blocker use is on the decline, due primarily to the introduction of prostaglandin analogues in the mid 1990s, which have a proven efficacy and a different side effect profile. ${ }^{12-15}$ In 1997 Gottfredsdottir et al ${ }^{21}$ showed that in the United States $91 \%$ of topical medications were beta-blockers during the period 1992-1994. In our study, topical beta-blocker use had reduced to $48.5 \%$ of those patients using antiglaucoma medication. It is unlikely however that beta-blocker use will discontinue, as they are very effective agents for pressure reduction. In our cohort 43 (25\%) of patients using topical medication required the use of both a beta-blocker and a prostaglandin analogue for intraocular pressure control.
Our study indicates that patients using topical betablockers have significantly lower resting pulse rates than those not using beta-blockers $(P=0.003)$, and is similar to previous studies. $^{3-8}$ This is probably due to the good bioavaliablity of topical beta-blockers ${ }^{22}$ therefore easily affecting the body systemically. An aspect of beta-blocker therapy, not widely considered in the literature, is the effect of combining oral and topical beta-blockers.

Schuman ${ }^{16}$ indicated that caution is required in combining topical and oral beta-blockers; however, this sample was taken from a randomised controlled trial and was not a cohort. Some studies have examined the use of multiple prescriptions. Gottfredsdottir et $a l^{21}$ showed that $84 \%$ of glaucoma patients in their practice were receiving one or more medications for systemic disease, with $8 \%$ using Atenolol, a systemic beta-blocker. Valuck et al ${ }^{23}$ showed that between 4.3 and $25.1 \%$ of glaucoma patients were prescribed systemic medication for a heart condition. Our cohort showed that the group combining the two medications was the only group in which the mean pulse rate was below that defined as bradycardic (mean 58 bpm, SD 10).

Unsurprisingly, there was an association to bradycardia if an oral beta-blocker is used in combination with a topical beta-blocker $(P=0.02)$ when compared to topical alone. However, the group using both topical and oral beta-blockers was also shown to be strongly associated with a bradycardia of below $50 \mathrm{bpm}$ when compared to the use of oral medication alone $(P=0.01)$. In our cohort, $27 \%$ of patients using both topical and oral beta-blockers were prescribed topical beta-blockers when already taking oral beta-blockers. There was however no association between hospital ophthalmologists or general practitioners commencing a concomitant beta-blocker and subsequent bradycardia, at $60 \mathrm{bpm}(P=0.57)$ or $50 \mathrm{bpm}(P=0.56)$. However, this highlights an important aspect of patient care, and without routine pulse rate monitoring, an up to date drug history, or knowledge of the concomitant effect of these drugs, ophthalmic and systemic beta-blocker use in patients appears potentially hazardous.

In order to clarify current practice, an anonymous questionnaire concerning pulse rate monitoring was given to a group of practicing consultant ophthalmologists, all with a special interest in glaucoma (Figure 3). The questionnaire was completed in $79 \%$ of cases $(n=11)$. Results indicate that $46 \%(n=5)$ of respondents measure pulse rates in glaucoma patients as part of their routine practice, with three stating 'before prescribing a beta-blocker', one stating 'at every visit', and one 'only if concerned'. All respondents stated that they routinely record the systemic medications taken by their glaucoma patients, but vary in their practice of this: $64 \%(n=7)$ 'at first visit only', 27\% $(n=3)$ 'every visit', 
Heart rate monitoring in glaucoma patients - questionnaire

$1 a$

Do you measure heart rates in your glaucoma patients as part of your practice?

\section{Yes / No}

1b If Yes do you measure:
On all patients at every visit

On all patients using beta-blockers at every visit.

Before prescribing a beta-blocker.

Only if concerned with cardiovascular function.

Other (please state)

2a Do you routinely review and record systemic medications in patients' medical records?

Yes / No

2b If Yes, when:

At first visit only

Every visit

Annually

Other (please state)

3 Do you consider that the addition of a topical beta-blocker to an existing systemic beta-blocker may adversely affect the patients heart rate?

Yes / No

Figure 3 Current practice questionnaire.

and 9\% $(n=1)$ 'annually'. When questioned as to their opinion whether the addition of a topical beta-blocker to an existing oral beta-blocker might adversely affect the patients' pulse rate $46 \%(n=5)$ stated 'no'.

It is of note that none of the patients not taking betablockers had a resting pulse rate of less than $50 \mathrm{bpm}$, and that the five patients with severe bradycardia were asymptomatic. Even so a clinically significant finding in one patient was found. Therefore routine pulse rate testing is advisable in patients using beta-blockers.

Recent advances in ophthalmology, such as the introduction of electronic patient management systems ${ }^{24}$ may well aid prescribers in the future by highlighting possible drug interactions, and therefore reduce the potential for harm to patients. However, despite new electronic management aids, and the reduction in topical beta-blocker usage, it remains crucial that the dangers of the concomitant use of topical and oral beta-blockers are born in mind when prescribing beta-blockers. Pulse rate monitoring and a review of ophthalmic medication is indicated in patients using beta-blocker therapy, as is good communication with the patients' general practitioner, to ensure that the health of patients is not compromised.

\section{References}

1 Zimmerman T. Topical ophthalmic beta-blockers: a comparative review. J Ocul Pharmacol 1993; 9(4): 373-384.

2 Airaksinen P, Valle O, Takki K, Klemetti A. Timolol treatment of chronic open angle glaucoma and ocular hypertension. A 2.5 year multicenter study. Graefes Arch Clin Exp Ophthalmol 1982; 219(2): 68-71.

3 Diggory P, Cassels-Brown A, Fernandez C. Topical betablockade with intrinsic sympathomimetic activity offers no advantage for the respiratory and cardiovascular function of elderly people. Age Ageing 1996; 25(6): 424-428. 
4 Hugues F, Matte J, Le Jeunne C, Salem A. Effects of betaadrenoceptors blocking eye drops in patients with chronic bronchitis. Therapie 1992; 47(3): 211-215.

5 Dorigo M, Cerin O, Fracasso G, Altafini R. Cardiovascular effects of befunolol, betaxolol and timolol eye drops. Int J Clin Pharmacol Res 1990; 10(3): 163-166.

6 Vuori M, Ali-Melkkila T, Kaila T, Iisalo E, Saari K. Plasma and aqueous humour concentrations and systemic effect of topical betaxolol and timolol in man. Acta Ophthalmol 1993; 71(2): 201-206.

7 Bauer K, Brunner-Ferber F, Distlerath L, Lippa E, Binkowitz B, Tilp P et al. Assessment of systemic effects of different ophthalmic beta-blockers in healthy volunteers. Clin Pharmacol Ther 1991; 49(6): 658-664.

8 Netland P, Weiss H, Stewart W, Cohen J, Nussbaum L. Cardiovascular effects of topical carteolol hydrochloride and timolol maleate in patients with ocular hypertension and primary open-angle glaucoma. Night study group. Am J Ophthalmol 1997; 123(4): 465-477.

9 Diggory P, Cassels-Brown A, Vail A, Hillman J. Randomised, controlled trial of spirometric changes in elderly people receiving timolol or betaxolol as initial treatment for glaucoma. Br J Ophthalmol 1998; 82(2): 146-149.

10 Diggory P, Cassels-Brown A, Vail A, Abbey L, Hillman J. Avoiding unsuspected respiratory side-effects of topical timolol with cardioselective or sympathomimetic agents. Lancet 1995; 345(8965): 1604-1606.

11 Diggory P, Heyworth P, Chau G, McKenzie S, Sharma A, Luke I. Improved lung function tests on changing from topical timolol non-selective beta-blockade impairs lung function tests in elderly patients. Eye 1993; 7(5): 661-663.

12 Chew P, Hung P, Aung T. Efficacy of latanoprost in reducing intraocular pressure in patients with primary angle-closure glaucoma. Surv Ophthalmol 2002; 47(Supp1): S125-S128.

13 Alm A, Stjernschantz J. Effects on intraocular pressure and side effects of $0.005 \%$ latanoprost applied once daily, evening or morning. A comparison with timolol. Scandinavian latanoprost study group. Ophthalmology 1995; 102(12): 1743-1752.
14 Camras C. Comparison of latanoprost and timolol in patients with ocular hypertension and glaucoma: a six month masked, multicenter trial in the United States. The United States latanoprost study group. Ophthalmology 1996; 103(1): 138-147.

15 Mastropasqua L, Carpineto P, Ciancaglini M, Gallenga P. A 12-month, randomized, double-masked study comparing latanoprost with timolol in pigmentary glaucoma. Ophthalmology 1999; 106(3): 550-555.

16 Schuman J. Effects of systemic beta-blocker therapy on the efficacy and safety of topical brimonidine and timolol. Brimonidine study groups 1 and 2. Ophthalmology 2000; 107(6): 1171-1177.

17 Gerber S, Cantor L, Brater C. Systemic drug interactions with topical glaucoma medications. Ophthalmology 1990; 35(3): 205-218.

18 Linkewich J, Herling I. Bradycardia and congestive heart failure associated with ocular timolol maleate. Am J Hosp Pharm 1981; 38(5): 699-701.

19 Pringle S, MacEwen C. Severe bradycardia due to interaction of timolol eye drops and verapamil. $\mathrm{Br}$ Med J 1987; 294: 155-156.

20 Darovic G. Hemodynamic Monitoring - Invasive and Noninvasive Clinical Application. WB Saunders: Philadelphia, 2002.

21 Gottfredsdottir M, Allingham R, Shields B. Physicians' guide to interactions between glaucoma and systemic medications. J Glaucoma 1997; 6(6): 377-383.

22 Korte J, Kaila T, Saari K. Systemic bioavailability and cardiopulmonary effects of $0.5 \%$ timolol eyedrops. Graefes Arch Clin Exp Ophthalmol 2002; 240(6): 430-435.

23 Valuck R, Perlman J, Anderson C, Wortman G. Coprescribing of medications used to treat obstructive lung disease, congestive heart failure and depression among users of topical beta blockers: estimates from three US veterans affairs medical centres. Pharmacoepidemiol Drug Saf 2001; 10(6): 511-516.

24 Vernon S. Electronic patient management systems in glaucoma. Glaucoma World 2002; 25: 11-15. 